\title{
Dynamic wormhole geometries in hybrid metric-Palatini gravity
}

\author{
Mahdi Kord Zangeneh ${ }^{1, \mathrm{a}} \mathbb{D}$, Francisco S. N. Lobo $^{2, \mathrm{~b}}{ }_{\overparen{C}}$ \\ ${ }_{1}^{1}$ Physics Department, Faculty of Science, Shahid Chamran University of Ahvaz, Ahvaz 61357-43135, Iran \\ ${ }^{2}$ Instituto de Astrofísica e Ciências do Espaço, Faculdade de Ciências da Universidade de Lisboa, Edifício C8, Campo Grande, 1749-016 Lisbon, \\ Portugal
}

Received: 3 January 2021 / Accepted: 13 March 2021 / Published online: 3 April 2021

(C) The Author(s) 2021

\begin{abstract}
In this work, we analyse the evolution of timedependent traversable wormhole geometries in a FriedmannLemaître-Robertson-Walker background in the context of the scalar-tensor representation of hybrid metric-Palatini gravity. We deduce the energy-momentum profile of the matter threading the wormhole spacetime in terms of the background quantities, the scalar field, the scale factor and the shape function, and find specific wormhole solutions by considering a barotropic equation of state for the background matter. We find that particular cases satisfy the null and weak energy conditions for all times. In addition to the barotropic equation of state, we also explore a specific evolving wormhole spacetime, by imposing a traceless energy-momentum tensor for the matter threading the wormhole and find that this geometry also satisfies the null and weak energy conditions at all times.
\end{abstract}

\section{Introduction}

The explanation of the accelerated expansion of the Universe is one of the most challenging problems in modern cosmology [1,2]. From the mathematical point of view, the simplest way to treat this problem is to consider the cosmological constant term [3]. Nonetheless, this model faces some difficulties such as the coincidence problem and the cosmological constant problem. The latter dictates a huge discrepancy between the observed values of the vacuum energy density and the theoretical large value of the zero-point energy suggested by quantum field theory [3]. There are some alternative models proposed to overcome these problems such as modified gravity [4-10], mysterious energy-momentum sources [11-13], such as quintessence [14-17] and $k$-essence [18-20] fields, and complex equations of state storing the missing energy of the dark side of the Universe [21,22]. In general, models with

\footnotetext{
a e-mail: mkzangeneh@scu.ac.ir

b e-mail: fslobo@fc.ul.pt (corresponding author)
}

varying dark energy candidates may be capable of overcoming all the mathematical and theoretical difficulties, however, the main underlying question is the origin of these terms. One proposal is to relate this behavior to the energy of quantum fields in vacuum through the holographic principle which allows us to reconcile infrared (IR) and ultraviolet (UV) cutoffs [23] (see Ref. [24] for a review on various attempts to model the dark side of the Universe).

However, an alternative to these dark energy models, as mentioned above, is modified gravity [4-10]. Here, one considers generalizations of the Hilbert-Einstein Lagrangian specific curvature invariants such as $R^{2}, R_{\mu \nu} R^{\mu \nu}$, $R_{\alpha \beta \mu \nu} R^{\alpha \beta \mu \nu}, \varepsilon^{\alpha \beta \mu \nu} R_{\alpha \beta \gamma \delta} R_{\mu \nu}^{\gamma \delta}$, etc. A popular theory that has attracted much attention is $f(R)$ gravity, where one may tackle the problem through several approaches, namely, the metric formalism [4-10], which considers that the metric is the fundamental field, or the Palatini formalism [25], where here one varies the action with respect to the metric and an independent connection. However, one may also consider a hybrid combination of these approaches that has recently been proposed, namely, the hybrid metric-Palatini gravitational theory [26], where the metric Einstein-Hilbert action is supplemented with a metric-affine (Palatini) correction term. The hybrid metric-Palatini theory has the ability to avoid several of the problematic issues that arise in the pure metric and Palatini formalisms. For instance, metric $f(R)$ gravity introduces an additional scalar degree of freedom, which must possess a low mass in order to be relevant for the large scale cosmic dynamics. However, the presence of such a low mass scalar field would influence the dynamics on smaller scales, such as at the level of the Solar System, and since these small scale effects remain undetected, one must resort to screening mechanisms [27,28]. Relative to the Palatini formalism, no additional degrees of freedom are introduced, as the scalar field is an algebraic function of the trace of the energymomentum tensor. It has been shown that this fact entails serious consequences for the theory, leading to the presence 
of infinite tidal forces on the surface of massive astrophysical type objects [25].

In this context, the hybrid metric-Palatini gravity theories were proposed initially in [26] in order to circumvent the above shortcomings in the metric and Palatini formalisms of $f(R)$ gravity. One of the main advantages of the hybrid metric-Palatini theory is that in its scalar-tensor representation a long-range force is introduced that automatically passes the Solar System tests, and thus no contradiction between the theory and the local measurements arise. In a cosmological context, it has also been shown that hybrid metric-Palatini gravity may also explain the cosmological epochs [29,30] (we refer the reader to [31-33] for more details). In fact, hybrid metric-Palatini gravity has attracted much attention recently, and has a plethora of applications, namely, in the galactic context [34-36], in cosmology [29,30,37-40], in braneworlds [41,42], black holes [4346], stellar solutions [47], cosmic strings [48,49], tests of binary pulsars [50], and wormholes [51,52], amongst others.

Here, we explore the possibility that evolving wormhole geometries may be supported by hybrid metric-Palatini gravity. These compact objects, which are theoretical shortcuts in spacetime, have been shown to be threaded by an exotic fluid that violates the null energy condition (NEC), at least for the static case $[53,54]$. However, it has been shown that evolving wormholes are able to satisfy the energy conditions in arbitrary but finite intervals of time $[55,56]$, contrary to their static counterparts $[57,58]$. One way to study this subject is to embed a wormhole in a Friedmann-LemaîtreRobertson-Walker (FLRW) metric, which permits the geometry to evolve in a cosmological background [59-75]. Due to the somewhat problematic nature of the energy condition violations, an important issue is whether traversable wormholes can be constructed from normal matter throughout the spacetime, or at least partially. Different wormhole structures have been explored from this point of view [74-79]. Indeed, it has been shown that in modified gravity, it is possible to impose that the matter threading the wormhole throat satisfies the energy conditions [80,81], and it is the higher order curvature terms that support these nonstandard wormhole geometries [82-87]. Furthermore, different wormhole structures have been studied extensively in the context of alternative theories of gravity [88-98] and also from different aspects [99-111].

In this paper, we study the evolution of traversable wormholes in a FLRW universe background in the scalar-tensor representation of hybrid metric-Palatini gravity. Furthermore, we explore the energy conditions for matter which threads these wormhole geometries. The paper is organized in the following manner: In Sect. 2, we briefly present the action and field equations of hybrid metric-Palatini gravity, we consider the spacetime metric and explore a barotropic equation of state for the background fluid. In Sect. 3, we anal- yse evolving traversable wormhole geometries for specific values of the barotropic equation of state parameter, as well as evolving wormholes with a traceless energy-momentum tensor (EMT), and study the energy conditions for the solutions obtained. Finally, in Sect. 4, we summary our results and conclude.

\section{Evolving wormholes in hybrid metric-Palatini gravity}

\subsection{Action and field equations}

Here, we briefly present the hybrid metric-Palatini gravitational theory. The action is given by

$S=\frac{1}{2 \kappa^{2}} \int d^{4} x \sqrt{-g}[R+f(\mathcal{R})]+S_{m}$,

where $\kappa^{2} \equiv 8 \pi G, R$ is the metric Ricci scalar, and the Palatini curvature is $\mathcal{R} \equiv g^{\mu \nu} \mathcal{R}_{\mu \nu}$, with the Palatini Ricci tensor, $\mathcal{R}_{\mu \nu}$, defined in terms of an independent connection, $\hat{\Gamma}_{\mu \nu}^{\alpha}$, given by

$\mathcal{R}_{\mu \nu} \equiv \hat{\Gamma}_{\mu \nu, \alpha}^{\alpha}-\hat{\Gamma}_{\mu \alpha, v}^{\alpha}+\hat{\Gamma}_{\alpha \lambda}^{\alpha} \hat{\Gamma}_{\mu \nu}^{\lambda}-\hat{\Gamma}_{\mu \lambda}^{\alpha} \hat{\Gamma}_{\alpha \nu}^{\lambda}$,

and $S_{m}$ is the matter action.

However, the scalar-tensor representation of hybrid metricPalatini gravity provides a theoretical framework which is easier to handle from a computational point of view [26], where the equivalent action to (1) is provided by

$S=\int \frac{d^{4} x \sqrt{-g}}{2 \kappa^{2}}\left[(1+\phi) R+\frac{3}{2 \phi} \partial_{\mu} \phi \partial^{\mu} \phi-V(\phi)\right]+S_{m}$.

Note a similarity with the action of the $w=-3 / 2$ BransDicke theory version of the Palatini approach to $f(R)$ gravity. However, the hybrid theory exhibits an important and subtle difference appearing in the scalar field-curvature coupling, which in the $w=-3 / 2$ Brans-Dicke theory is of the form $\phi R[26]$.

By varying the action (3) with respect to the metric provides the following gravitational field equation

$G_{\mu \nu}=\kappa^{2}\left(\frac{1}{1+\phi} T_{\mu \nu}+T_{\mu \nu}^{(\phi)}\right)$

where $T_{\mu \nu}$ is the standard matter energy-momentum tensor, and

$$
\begin{aligned}
T_{\mu \nu}^{(\phi)}= & \frac{1}{\kappa^{2}} \frac{1}{1+\phi}\left[\nabla_{\mu} \nabla_{\nu} \phi-\frac{3}{2 \phi} \nabla_{\mu} \phi \nabla_{\nu} \phi\right. \\
& \left.+\left(\frac{3}{4 \phi} \nabla_{\lambda} \phi \nabla^{\lambda} \phi-\square \phi-\frac{1}{2} V\right) g_{\mu \nu}\right],
\end{aligned}
$$


is the energy-momentum tensor of the scalar field of the theory.

Varying the action with respect to the scalar field yields the following second-order differential equation,

$$
-\square \phi+\frac{1}{2 \phi} \partial_{\mu} \phi \partial^{\mu} \phi+\frac{\phi\left[2 V-(1+\phi) V_{\phi}\right]}{3}=\frac{\phi \kappa^{2}}{3} T .
$$

The above equation shows that in hybrid metric-Palatini gravity the scalar field is dynamical. This represents an important and interesting difference with respect to the standard Palatini case [25].

\subsection{Spacetime metric}

The metric of a time-dependent wormhole geometry is given by

$$
d s^{2}=-e^{2 \Phi(r)} d t^{2}+a^{2}(t)\left[\frac{d r^{2}}{1-b(r) / r}+r^{2} d \Omega^{2}\right],
$$

where $\Phi(r)$ and $b(r)$, respectively, are the redshift and shape functions, which are $r$-dependent, and $a(t)$ is the timedependent scale factor, and $d \Omega^{2}=d \theta^{2}+\sin ^{2} \theta d \varphi^{2}$ is the linear element of the unit sphere.

To correspond to a wormhole solution, the shape function has the following restrictions [53]: (i) $b\left(r_{0}\right)=r_{0}$, where $r_{0}$ is the wormhole throat, corresponding to a minimum radial coordinate, (ii) $b(r) \leq r$, and (iii) the flaring-out condition given in the form $r b^{\prime}(r)-b(r)<0$. The latter condition is the fundamental ingredient in wormhole physics, as taking into account the Einstein field equation, one verifies that this flaring-out condition imposes the violation of the NEC. In fact, it violates all of the pointwise energy conditions $[53,54$, $57,58,112]$. In order to avoid the presence of event horizons, so that the wormhole is traversable, one also imposes that the redshift function, $\Phi(r)$, be finite everywhere. However, throughout this work, we consider a zero redshift function, $\Phi=0$, which simplifies the calculations significantly.

\subsection{Evolving embedding analysis}

It is also interesting to explore how the time evolution of the scale factor affects the wormhole itself. To this effect, in the following analysis, we will follow closely the analysis outlined in Refs. [53,59,75], and analyse how the (effective) radius of the throat or length of the wormhole changes with time. As mentioned above, throughout this paper, we consider $\Phi=0$ for simplicity. Thus, in order to analyse the time-dependent dynamic wormhole geometry, we need to choose a specific $b(r)$ to provide a reasonable wormhole at $t=0$, which is assumed to be the onset of the evolution. Note that the radial proper length between any two points
$A$ and $B$, through the wormhole, at any $t=$ const is given by $l(t)= \pm a(t) \int_{r_{A}}^{r_{B}}(1-b / r)^{-1 / 2} d r$, which is merely the initial radial proper separation multiplied by the scale factor.

Now, to analyse how the "wormhole" form of the metric is maintained throughout the evolution, we consider a $t=$ const and $\theta=\pi / 2$ slice of the spacetime (7). Thus, the metric reduces to

$d s^{2}=\frac{a^{2}(t) d r^{2}}{1-b(r) / r}+a^{2}(t) r^{2} d \varphi^{2}$,

and is embedded in a flat 3-dimensional Euclidean space given by

$d s^{2}=d \bar{z}^{2}+d \bar{r}^{2}+\bar{r}^{2} d \varphi^{2}$.

Comparing the angular coefficients, yields

$$
\begin{aligned}
\bar{r} & =\left.a(t) r\right|_{t=\text { const }}, \\
d \bar{r}^{2} & =\left.a^{2}(t) d r^{2}\right|_{t=\text { const }} .
\end{aligned}
$$

Note, that when considering derivatives, these relations do not represent a "coordinate transformation", but a "rescaling" of the radial coordinate $r$ along each $t=$ constant slice.

The "wormhole" form of the metric will be preserved, with respect to the $\bar{z}, \bar{r}, \varphi$ coordinates, if the embedded slice has the following metric

$d s^{2}=\frac{d \bar{r}^{2}}{1-\bar{b}(\bar{r}) / \bar{r}}+\bar{r}^{2} d \varphi^{2}$,

and the shape functions $\bar{b}(\bar{r})$ has a minimum at a specific $\bar{b}\left(\bar{r}_{0}\right)=\bar{r}_{0}$. Now, the embedded slice (8) can be readily rewritten in the form of Eq. (12) by using Eqs. (10) and (11), and the following relation

$\bar{b}(\bar{r})=a(t) b(r)$.

Thus, the evolving wormhole will have the same overall shape and size and relative to the $\bar{z}, \bar{r}, \varphi$ coordinate system, as the initial wormhole had relative to the initial $z, r, \varphi$ embedding space coordinate system. In addition to this, using Eqs. (9) and (12), one deduces that

$$
\frac{d \bar{z}}{d \bar{r}}= \pm\left(\frac{\bar{r}}{\bar{b}(\bar{r})}-1\right)^{-1 / 2}=\frac{d z}{d r}
$$

which implies

$$
\begin{aligned}
\bar{z}(\bar{r}) & = \pm \int \frac{d \bar{r}}{(\bar{r} / \bar{b}(\bar{r})-1)^{1 / 2}} \\
& = \pm a(t) \int\left(\frac{r-b}{b}\right)^{-1 / 2} d r \\
& = \pm a(t) z(r) .
\end{aligned}
$$


Thus, the relation between the initial embedding space at $t=0$ and the embedding space at any time $t$ is, from Eqs. (11) and (15)

$d s^{2}=d \bar{z}^{2}+d \bar{r}^{2}+\bar{r}^{2} d \varphi^{2}=a^{2}(t)\left[d z^{2}+d r^{2}+r^{2} d \varphi^{2}\right]$.

The wormhole will always remain the same size, relative to the $\bar{z}, \bar{r}, \phi$ coordinate system, as the scaling of the embedding space compensates for the evolution of the wormhole. Nevertheless, the wormhole will change size relative to the initial $t=0$ embedding space.

We also note that the "flaring out condition" for the evolving wormhole is given by

$\frac{d^{2} \bar{r}(\bar{z})}{d \bar{z}^{2}}>0$

at or near the throat, so that taking into account Eqs. (10), (11), (13), and (14), it follows that

$\frac{d^{2} \bar{r}(\bar{z})}{d \bar{z}^{2}}=\frac{1}{a(t)} \frac{b-b^{\prime} r}{2 b^{2}}=\frac{1}{a(t)} \frac{d^{2} r(z)}{d z^{2}}>0$,

at or near the throat. Using Eqs. (10), (13), and

$\bar{b}^{\prime}(\bar{r})=\frac{d \bar{b}}{d \bar{r}}=b^{\prime}(r)=\frac{d b}{d r}$,

the right-hand side of Eq. (18), relative to the $\bar{z}, \bar{r}, \phi$ coordinate system, may be written as

$\frac{d^{2} \bar{r}(\bar{z})}{d \bar{z}^{2}}=\left(\frac{\bar{b}-\bar{b}^{\prime} \bar{r}}{2 \bar{b}^{2}}\right)>0$,

at or near the throat. Thus, we have verified that the flaring out condition (20), using the barred coordinates, has the same form as for the static wormhole.

\subsection{Gravitational field equations}

We also consider an anisotropic matter energy-momentum tensor given by $T_{v}^{\mu}=\operatorname{diag}(-\rho,-\tau, p, p)$, where $\rho, \tau$ and $p$ are the energy density, the radial tension (which is equivalent to a negative radial pressure) and the tangential pressure, respectively. Using the metric (7), the gravitational field equations (4) provide the following energy-momentum profile:

$$
\begin{aligned}
& \rho(t, r)=\left(3 H^{2}+\frac{b^{\prime}}{r^{2} a^{2}}\right)(1+\phi)+3 \dot{\phi} H+\frac{3 \dot{\phi}^{2}}{4 \phi}-\frac{1}{2} V(\phi), \\
& \tau(t, r)=\left(H^{2}+2 \frac{\ddot{a}}{a}+\frac{b}{r^{3} a^{2}}\right)(1+\phi) \\
& +2 \dot{\phi} H-\frac{3 \dot{\phi}^{2}}{4 \phi}+\ddot{\phi}-\frac{1}{2} V(\phi),
\end{aligned}
$$

$$
\begin{aligned}
& p(t, r)=\left(-H^{2}-2 \frac{\ddot{a}}{a}-\frac{r b^{\prime}-b}{2 r^{3} a^{2}}\right)(1+\phi) \\
& -2 \dot{\phi} H+\frac{3 \dot{\phi}^{2}}{4 \phi}-\ddot{\phi}+\frac{1}{2} V(\phi),
\end{aligned}
$$

respectively, where $H=\dot{a} / a$ and $\phi=\phi(t)$, the overdot and prime denote derivatives with respect to $t$ and $r$, respectively, and for notational simplicity, we have considered $\kappa=1$. One recovers the standard field equations of the Morris-Thorne wormhole [53], by fixing the scale factor $a$ to unity and excluding the background time-dependent evolution and the scalar field $\phi$ contribution.

Furthermore, we will also analyse the null and weak energy conditions for the solutions obtained below. The weak energy condition (WEC) is defined as $T_{\mu \nu} u^{\mu} u^{\nu} \geq 0$, where $u^{\mu}$ is a timelike vector, and is expressed in terms of the energy density $\rho$, radial tension $\tau$ and tangential pressure $p$ as $\rho \geq 0$, $\rho-\tau \geq 0$ and $\rho+p \geq 0$, respectively. The last two inequalities, i.e., $\rho-\tau \geq 0$ and $\rho+p \geq 0$ correspond to the NEC, which is defined as $T_{\mu \nu} k^{\mu} k^{\nu} \geq 0$, where $k^{\mu}$ is any null vector.

The scalar field equation of motion (6) yields

$\ddot{\phi}+3 \dot{\phi} H-\frac{\dot{\phi}^{2}}{2 \phi}-\frac{1}{3} \phi\left[T+(1+\phi) \frac{d V}{d \phi}-2 V(\phi)\right]=0$,

where the trace of the energy-momentum tensor $T=$ $T(t, r)=T_{\mu}^{\mu}=-\rho-\tau+2 p$ is given by

$$
\begin{gathered}
T(t, r)=-2\left(\frac{b^{\prime}}{a^{2} r^{2}}+3 H^{2}+3 \frac{\ddot{a}}{a}\right)(1+\phi) \\
-3 \ddot{\phi}+\frac{3 \dot{\phi}^{2}}{2 \phi}-9 \dot{\phi} H+2 V(\phi) .
\end{gathered}
$$

In order to solve the differential equation (24) for $\phi(t)$, the trace $T(t, r)$, given by Eq. (25), should be independent of $r$. This condition leads to $b^{\prime} / r^{2}=C H_{0}^{2}$ where $H_{0}$ is the present value of the Hubble parameter and $C$ is an arbitrary dimensionless constant. Thus, the shape function is given by $b(r)=r_{0}+C H_{0}^{2}\left(r^{3}-r_{0}^{3}\right) / 3$, which satisfies $b\left(r_{0}\right)=r_{0}$, and the flaring-out condition at the throat imposes $\mathrm{CH}_{0}^{2} r_{0}^{2}<1$.

To solve Eq. (24) numerically for $\phi$, it is useful to rewrite it in terms of dimensionless functions of the scale factor $a$. Thus, we consider the definitions:

$$
\begin{aligned}
& \ddot{\phi}=\ddot{a} \phi^{\prime}(a)+\dot{a}^{2} \phi^{\prime \prime}(a), \quad \dot{\phi}=\dot{a} \phi^{\prime}(a), \\
& \ddot{a}=H \dot{a}+\dot{H} a, \quad \dot{H}=\dot{a} H^{\prime}(a), \quad \dot{a}=H a .
\end{aligned}
$$

Note that in the above definitions, the prime denotes a derivative with respect to the scale factor. In addition to this, we consider $U=V / 3 H_{0}^{2}$ and $E=H / H_{0}$, so that Eq. (24) finally takes the following form

$$
\phi^{\prime \prime}(a)-\frac{\phi^{\prime 2}}{2 \phi(a)}+\frac{4\left(a \phi^{\prime}(a)+\phi(a)\right)}{a^{2}}+\frac{E^{\prime}(a) \phi^{\prime}(a)}{E(a)}
$$


$+\frac{2 C \phi(a)}{3 a^{4} E(a)^{2}}+\frac{2 \phi(a) E^{\prime}(a)}{a E(a)}-\frac{\phi(a)}{a^{2} E(a)^{2}} \frac{d U}{d \phi}=0$.

\subsection{Barotropic equation of state}

To solve the differential equation (26) for $\phi(a)$, we need to deduce $E$. To this effect, it is also useful to define the following background quantities:

$\rho_{b}(t)=3 H^{2}, \quad \tau_{b}(t)=H^{2}+2 \frac{\ddot{a}}{a}$,

and consider a background barotropic equation of state given by $\tau_{b}=-\omega_{b} \rho_{b}$. From this condition, we find $E=$ $a^{-3\left(\omega_{b}+1\right) / 2}$. One could solve this equation of state for scale factor as a function of the time coordinate $t$, which yields $a(t) \propto t^{2 / 3\left(1+\omega_{b}\right)}$. For $\omega_{b}=-1$, the scale factor takes the exponential form in terms of $t$. Furthermore, we set $C=0$ which satisfies the flaring-out condition at the throat (see discussion below Eq. (25)), and in addition simplifies the solution, so that $b(r)=r_{0}$. Thus, taking into these conditions and using the dimensionless definitions given above, Eqs. (21)-(23) lead to the following relations

$$
\begin{gathered}
\frac{\rho}{3 H_{0}^{2}}=\frac{1+\phi(a)}{a^{3\left(\omega_{b}+1\right)}}+\frac{\phi^{\prime}(a)}{a^{3 \omega_{b}+2}}+\frac{\phi^{\prime 2}(a)}{4 a^{3 \omega_{b}+1} \phi(a)}-\frac{1}{2} U(\phi), \\
\frac{\rho-\tau}{3 H_{0}^{2}}=\left(\frac{\omega_{b}+1}{a^{3\left(\omega_{b}+1\right)}}-\frac{r_{0}}{3 a^{2} H_{0}^{2} r^{3}}\right)(1+\phi(a)) \\
\quad+\frac{\left(\omega_{b}+1\right) \phi^{\prime}(a)}{2 a^{3 \omega_{b}+2}}+\frac{\phi^{\prime 2}(a)}{2 a^{3 \omega_{b}+1} \phi(a)}-\frac{\phi^{\prime \prime}(a)}{3 a^{3 \omega_{b}+1}}, \\
\frac{\rho+p}{3 H_{0}^{2}}=\left(\frac{\omega_{b}+1}{a^{3\left(\omega_{b}+1\right)}}+\frac{r_{0}}{6 a^{2} H_{0}^{2} r^{3}}\right)(1+\phi(a)) \\
+\frac{\left(\omega_{b}+1\right) \phi^{\prime}(a)}{2 a^{3 \omega_{b}+2}}+\frac{\phi^{2}(a)}{2 a^{3 \omega_{b}+1} \phi(a)}-\frac{\phi^{\prime \prime}(a)}{3 a^{3 \omega_{b}+1}},
\end{gathered}
$$

respectively. It is interesting to note that $\rho$ is independent of the radial coordinate $r$. To keep the terms dimensionless, we will consider the wormhole throat as $r_{0}=A H_{0}^{-1}$, where $A$ is a dimensionless constant. In what follows, we set $A$ to unity.

In the following, we shall consider specific solutions for the parameters $\omega_{b}=(-1,1 / 3,0)$, respectively. These parameters range tentatively correspond to the inflationary, radiation and matter epochs.

\section{Specific evolving wormhole solutions and the energy conditions}

In this section, we analyse evolving traversable wormhole geometries for the specific parameters $\omega_{b}=(-1,1 / 3,0)$ considered above, as well as evolving wormholes with a traceless EMT, and study the NEC and WEC for the solutions obtained.
Before going on further, we would like to comment on the potential for the scalar field $U(\phi)$ and its effects on the results. As Eqs. (29) and (30) (and (21)-(23)) show, the quantities $\rho-\tau$ and $\rho+p$ (and consequently the NEC) are independent of the scalar field potential. However, it affects the behavior of $\rho$. We will consider a power law potential for scalar field $U(\phi)=\phi^{\alpha}$. There are some reasonable choice for $\alpha$, such as $\alpha=2$ and $\alpha=4$, inspired by a mass term and the Higgs potential, respectively. In fact, we emphasize that all the results below hold qualitatively for potentials of the form $\phi^{2}$ and $\phi^{4}$. For the sake of economy, we will present the results for the quadratic case, $U(\phi)=\phi^{2}$. In addition to this, to verify that other choices for $\alpha$ could also present reliable results, we consider the specific choice $\alpha=0.5$ in one of the cases.

\subsection{Specific case: $\omega_{b}=-1$}

Here, we consider the specific case $\omega_{b}=-\tau_{b} / \rho_{b}=-1$ with $U(\phi)=V(\phi) / 3 H_{0}^{2}=\phi^{0.5}$. Figure 1a depicts the behaviors of $\phi$ and $\phi^{\prime}$ with respect to the redshift $z(=1 / a-1)$. In Fig. 2, the behaviors of $\rho, \rho-\tau$ and $\rho+p$ versus $z$ for different values of $r$ are shown. As one can see, $\rho-\tau$ is negative throughout the entire evolution. We emphasize that we have imposed different possible initial values and obtained the same result. Note that one may justify this outcome in the following manner. For this case with $\omega_{b}=-1$, Eq. (29) reduces to

$$
\frac{\rho-\tau}{3 H_{0}^{2}}=-\frac{(1+\phi(a)) r_{0}}{3 a^{2} H_{0}^{2} r^{3}}+a^{2}\left(\frac{\phi^{\prime 2}(a)}{2 \phi(a)}-\frac{\phi^{\prime \prime}(a)}{3}\right) .
$$

Since $a \leq 1$, the term with a coefficient $1 / a^{2}$ is dominant. This term is therefore negative, rendering $\rho-\tau$ negative. Thus, for this specific case, both the NEC and the WEC are violated.

\subsection{Specific case: $\omega_{b}=1 / 3$}

Figure $1 \mathrm{~b}$ illustrates the behavior of $\phi$ and $\phi^{\prime}$ versus $z$ for the specific case $\omega_{b}=1 / 3$. For this case, $E=a^{-2}, a(t) \propto t^{1 / 2}$ and we consider $U(\phi)=\phi^{2}$. The behaviors of $\rho, \rho-\tau$ and $\rho+p$ with respect to the redshift $z$ for different values of $r$ are displayed in Fig. 3, which shows explicitly that these quantities decrease as time evolves. However, they remain positive at all times and consequently the NEC and WEC are always satisfied. This occurs for the wormhole throat as well as for other wormhole radii. It is interesting to note that at a specified time/redshift, the quantity $\rho-\tau$ increases for increasing values of the radius, and the minimum value corresponds to the throat, as depicted by Fig. 3b. On the other hand, $\rho+p$ decreases for increasing values of the radius, and has a maximum at the throat (Fig. 3c). 


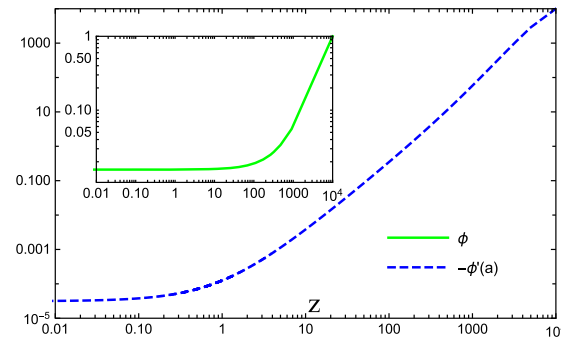

(a) Specific case of $\omega_{b}=-1$ with $U(\phi)=\phi^{0.5}$

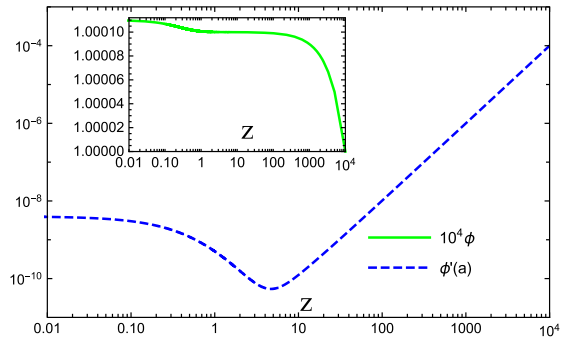

(b) Specific case of $\omega_{b}=1 / 3$ with $U(\phi)=\phi^{2}$

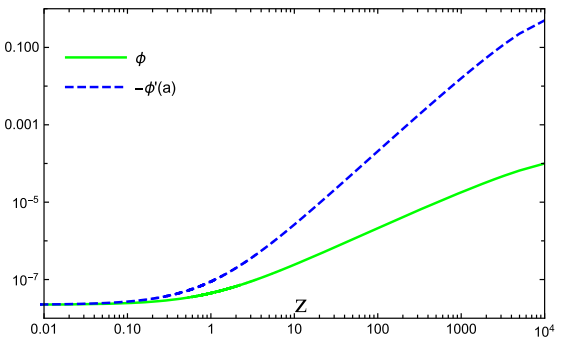

(c) Specific case of $\omega_{b}=0$ with $U(\phi)=\phi^{2}$

Fig. 1 The behaviors of $\phi$ and $\phi^{\prime}(a)$ vs $z$ for the specific cases of $\omega_{b}=-1, \omega_{b}=1 / 3$, and $\omega_{b}=0$, respectively. Note that both horizontal and vertical axes are logarithmic

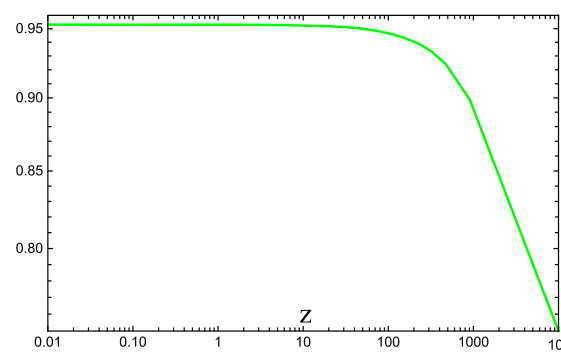

(a) $\rho / 3 H_{0}^{2}$ vs $z$

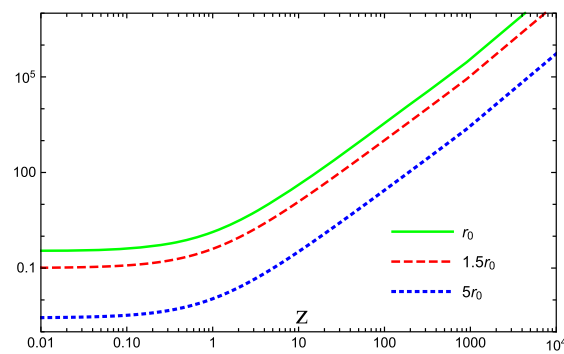

(b) $|\rho-\tau| / 3 H_{0}^{2}$ vs $z$

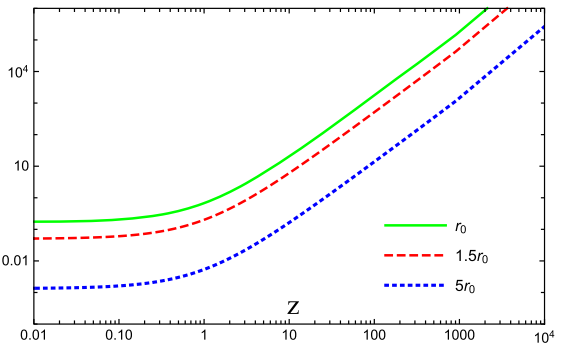

(c) $(\rho+p) / 3 H_{0}^{2}$ vs $z$

Fig. 2 The behaviors of $\rho, \rho-\tau$ (which is negative) and $\rho+p$, respectively, versus $z$ for different values of $r$ for $\omega_{b}=-1$ with $U(\phi)=\phi^{0.5}$. Note that both horizontal and vertical axes are logarithmic, except for the vertical axis of Fig. 2a

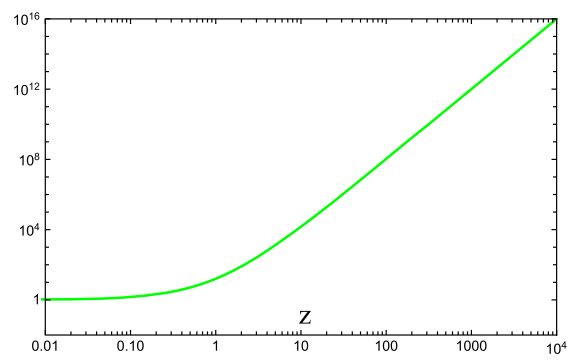

(a) $\rho / 3 H_{0}^{2}$ vs $z$

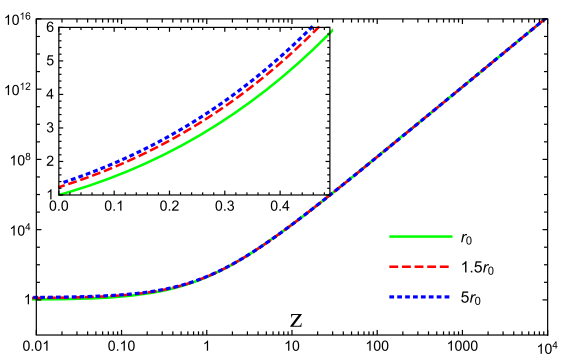

(b) $(\rho-\tau) / 3 H_{0}^{2}$ vs $z$

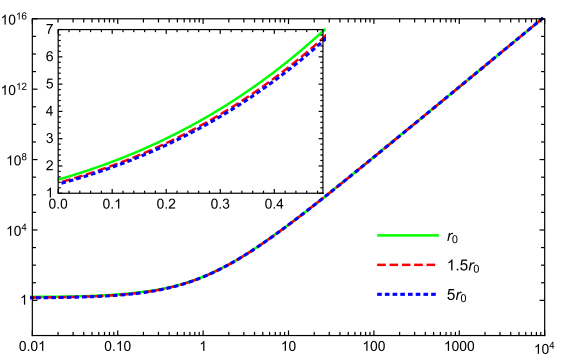

(c) $(\rho+p) / 3 H_{0}^{2}$ vs $z$

Fig. 3 The behaviors of $\rho, \rho-\tau$ and $\rho+p$, respectively, versus $z$ for different values of $r$ in the specific case of $\omega_{b}=1 / 3$ with $U(\phi)=\phi^{2}$. Note that both the horizontal and vertical axes are logarithmic

\subsection{Specific case: $\omega_{b}=0$}

Here we intend to study the energy conditions for the case $\omega_{b}=0$, for which $E=a^{-3 / 2}$ and $a(t) \propto t^{2 / 3}$. One can verify the behaviors of the scalar field $\phi$ and its derivative with respect to $a$ as functions of the redshift $z$ in Fig. 1c, where we consider the power law potential corresponding to scalar field as $U(\phi)=\phi^{2}$. The energy conditions are depicted in Fig. 4, where one verifies that $\rho, \rho-\tau$ and $\rho+p$ are positive at all times and for different values of spatial coordinate $r$. These quantities are also decreasing (increasing) functions with respect to time (redshift). Thus, the NEC and WEC are always satisfied for all wormhole radii including the wormhole throat. The behaviors of $\rho-\tau$ and $\rho+p$ at a specified time/redshift are analogous to the previous case with $\omega_{b}=1 / 3$, namely, the quantity $\rho-\tau(\rho+p)$ increases (decreases) for increasing values of the radius (see Fig. 4b, c).

\subsection{Wormholes with traceless EMT}

In this subsection, we consider the traceless energy-momentum tensor, with $T=-\rho-\tau+2 p=0$, which taking into account the dimensionless quantities defined above, provides the following differential equation

$$
\begin{gathered}
-3 a^{2} E(a)^{2}\left[\phi^{\prime \prime}(a)-\frac{\phi^{\prime}(a)^{2}}{2 \phi(a)}\right]-3 a E(a)\left[a E^{\prime}(a)\right. \\
+4 E(a)] \phi^{\prime}(a)-6 E(a)\left[a E^{\prime}(a)+2 E(a)\right]
\end{gathered}
$$




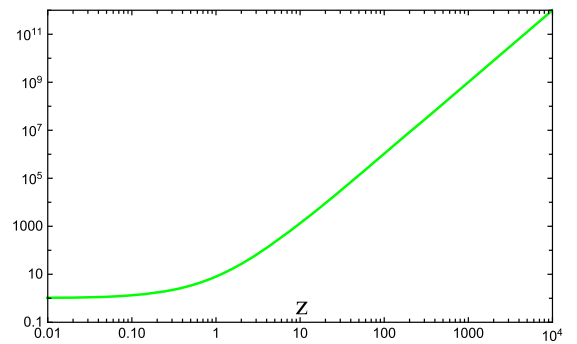

(a) $\rho / 3 H_{0}^{2}$ vs $z$

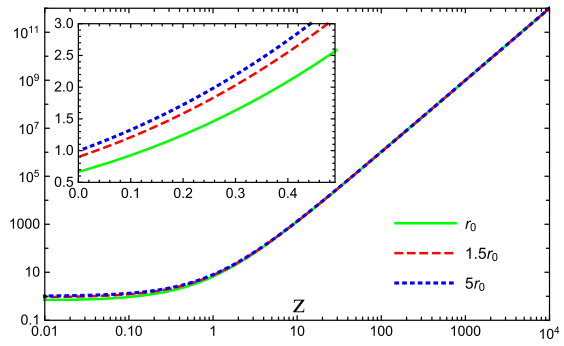

(b) $(\rho-\tau) / 3 H_{0}^{2}$ vs $z$

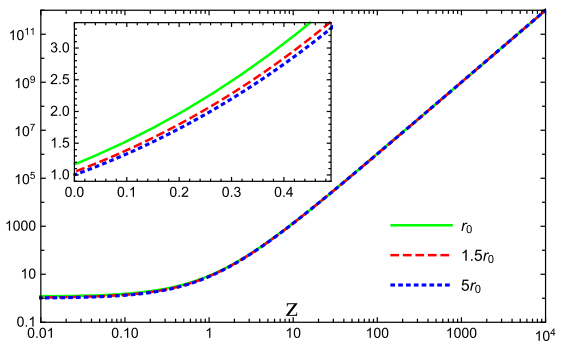

(c) $(\rho+p) / 3 H_{0}^{2}$ vs $z$

Fig. 4 The behaviors of $\rho, \rho-\tau$ and $\rho+p$, respectively, versus $z$ for different values of $r$ in the specific case of $\omega_{b}=0$ with $U(\phi)=\phi^{2}$. Both horizontal and vertical axes are logarithmic

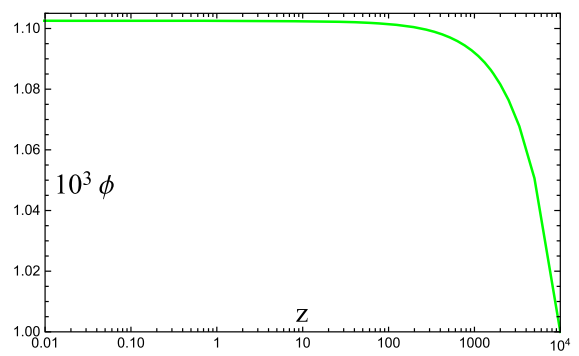

(a) $\phi$ vs $z$

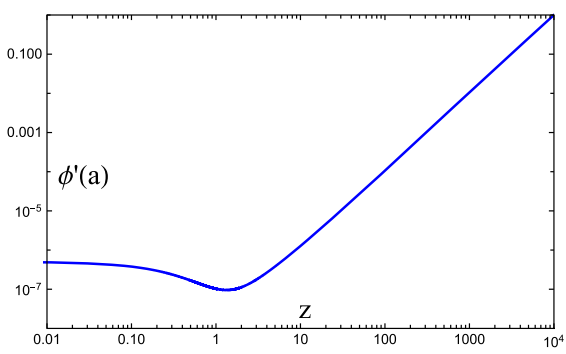

(b) $\phi^{\prime}(a)$ vs $z$

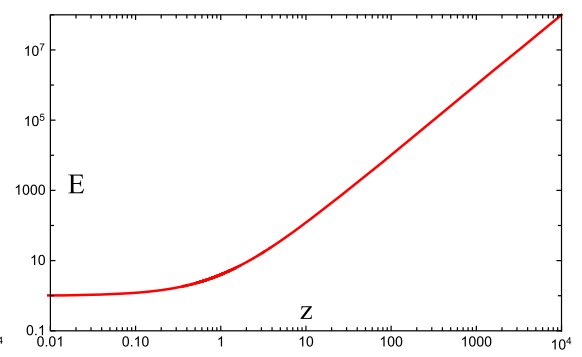

(c) $E$ vs $z$

Fig. 5 The behaviors of $\phi, \phi^{\prime}(a)$ and $E$ vs $z$ for the traceless EMT case with $U(\phi)=\phi^{2}$. Note that both horizontal and vertical axes are logarithmic, except for the vertical axis of Fig. 5a. See the text for more details

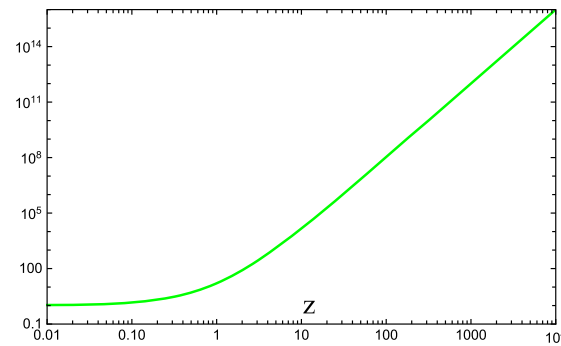

(a) $\rho / 3 H_{0}^{2}$ vs $z$

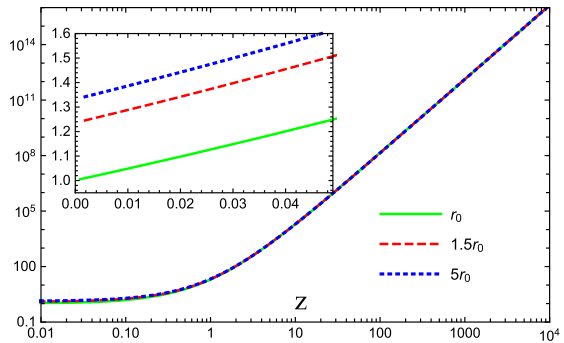

(b) $(\rho-\tau) / 3 H_{0}^{2}$ vs $z$

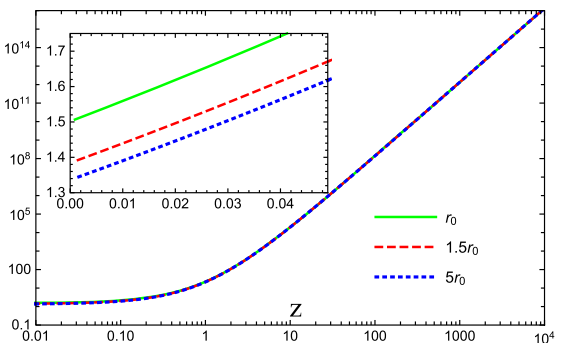

(c) $(\rho+p) / 3 H_{0}^{2}$ vs $z$

Fig. 6 The behaviors of $\rho, \rho-\tau$ and $\rho+p$, respectively, versus $z$ for different values of $r$ for a wormhole with traceless EMT with $U(\phi)=\phi^{2}$. Both horizontal and vertical axes are logarithmic

$$
\times[1+\phi(a)]+6 U(\phi)=0 .
$$

Here, we solve the system of coupled differential equations (26) and (32), for $\phi$ and $E$, numerically. The behaviors of $\phi$, $\phi^{\prime}$ and $E$ versus $z$, taking into account the choice $U(\phi)=$ $\phi^{2}$, are shown in Fig. 5. Note that $E$ tends to unity at the present time $(z \rightarrow 0)$, as expected (Fig. 5c). In Fig. 6, the behaviors of $\rho, \rho-\tau$ and $\rho+p$, with respect to $z$ for different values of $r$ are displayed. Note that $\rho, \rho-\tau$ and $\rho+p$ are decreasing (increasing) functions of time (redshift) and are positive for all radii, as time evolves. Thus, the NEC and WEC are satisfied at all times. Moreover, as shown in Fig. 6b, $\mathrm{c}$, the qualitative behaviors of $\rho-\tau$ and $\rho+p$ with respect to $r$ is analogous to the previous two cases, namely, $\omega_{b}=1 / 3$ and $\omega_{b}=0$, where the quantity $\rho-\tau(\rho+p)$ has a minimum (maximum) value at throat.

\section{Discussion and conclusion}

In the present paper, we have studied the evolution of dynamic traversable wormhole geometries in a FLRW background in the context of hybrid metric-Palatini gravity. This theory, which recently attracted much attention, consists of a hybrid combination of metric and Palatini terms and is capable of avoiding several of the problematic issues associated to each of the metric or Palatini formalisms (for more details, we refer the reader to Refs. [31-33]). For the evolving wormholes, we presented the components of the energymomentum tensor that supports these geometries in terms of the model's functions, namely, the scalar field, the scale factor and the shape function (we considered a zero redshift function, for simplicity). Furthermore, we found specific worm- 
hole solutions by considering a barotropic equation of state for the background matter, i.e., $\tau_{b}=-\omega_{b} \rho_{b}$, and considered particular equation of state parameters.

More specifically, we showed that for the specific cases of $\omega_{b}=1 / 3$ and $\omega_{b}=0$, the entire wormhole matter satisfies the NEC and WEC for all times. The latter cases are similar to the wormhole geometries analysed in the presence of pole dark energy [75], however, there the WEC is violated at late times, i.e., the energy density becomes negative. Thus, the present results outlined in this work strengthen the varying dark energy models and may suggest that hybrid metricPalatini gravity is a rather more promising model to explore. In addition to the barotropic equation of state, we also studied evolving wormhole geometries supported by the matter with a traceless EMT. For this specific geometry, we discovered that both the NEC and the WEC are satisfied at all times as well. These results are extremely promising as they build on previous work that consider that the energy conditions may be satisfied in specific flashes of time [55,56].

An interesting astrophysical observational aspect on how to detect these wormholes would be to analyse the physical properties and characteristics of matter forming thin accretion disks around the wormhole geometries analysed in this work, much in the spirit of the analysis carried out in Refs. [113-116]. In fact, specific signatures could appear in the electromagnetic spectrum, thus leading to the possibility of distinguishing these wormhole geometries by using astrophysical observations of the emission spectra from accretion disks. This would be an interesting avenue of research to explore.

Acknowledgements We thank the referees for the constructive comments that helped us to significantly improve the paper. MKZ would like to thank Shahid Chamran University of Ahvaz, Iran for supporting this work. FSNL acknowledges support from the Fundação para a Ciência e a Tecnologia (FCT) Scientific Employment Stimulus contract with reference CEECIND/04057/2017, and thanks funding from the research Grants no. PTDC/FIS-OUT/29048/2017, no. CERN/FISPAR/0037/2019 and no. UID/FIS/04434/2020.

Data Availability Statement This manuscript has no associated data or the data will not be deposited. [Authors' comment: This is a theoretical study and no experimental data has been listed.]

Open Access This article is licensed under a Creative Commons Attribution 4.0 International License, which permits use, sharing, adaptation, distribution and reproduction in any medium or format, as long as you give appropriate credit to the original author(s) and the source, provide a link to the Creative Commons licence, and indicate if changes were made. The images or other third party material in this article are included in the article's Creative Commons licence, unless indicated otherwise in a credit line to the material. If material is not included in the article's Creative Commons licence and your intended use is not permitted by statutory regulation or exceeds the permitted use, you will need to obtain permission directly from the copyright holder. To view a copy of this licence, visit http://creativecomm ons.org/licenses/by/4.0/.

Funded by SCOAP ${ }^{3}$.

\section{References}

1. S. Perlmutter et al. (Supernova Cosmology Project), Measurements of $\Omega$ and $\Lambda$ from 42 high redshift supernovae. Astrophys. J. 517, 565-586 (1999). arXiv:astro-ph/9812133

2. A.G. Riess et al. (Supernova Search Team), Observational evidence from supernovae for an accelerating universe and a cosmological constant. Astron. J. 116, 1009-1038 (1998). arXiv:astro-ph/9805201

3. S.M. Carroll, The cosmological constant. Living Rev. Relativ. 4, 1 (2001). arXiv:astro-ph/0004075

4. S. Nojiri, S.D. Odintsov, Unified cosmic history in modified gravity: from $\mathrm{F}(\mathrm{R})$ theory to Lorentz non-invariant models. Phys. Rep. 505, 59-144 (2011). arXiv:1011.0544 [gr-qc]

5. T.P. Sotiriou, V. Faraoni, $f(R)$ theories of gravity. Rev. Mod. Phys. 82, 451-497 (2010). arXiv:0805.1726 [gr-qc]

6. S. Capozziello, M. De Laurentis, Extended theories of gravity. Phys. Rep. 509, 167-321 (2011). arXiv:1108.6266 [gr-qc]

7. P. Avelino, T. Barreiro, C.S. Carvalho, A. da Silva, F.S.N. Lobo, P. Martin-Moruno, J.P. Mimoso, N.J. Nunes, D. Rubiera-Garcia, D. Saez-Gomez et al., Unveiling the dynamics of the Universe. Symmetry 8(8), 70 (2016). arXiv:1607.02979 [astro-ph.CO]

8. F. S. N. Lobo, The dark side of gravity: Modifed theories of gravity, Dark Energy-Current Advances and Ideas, 173-204 (2009), Research Signpost. arXiv:0807.1640 [gr-qc]

9. K. Bamba, S.D. Odintsov, Inflationary cosmology in modified gravity theories. Symmetry 7(1), 220-240 (2015). arXiv:1503.00442 [hep-th]

10. S. Nojiri, S.D. Odintsov, V.K. Oikonomou, Modified gravity theories on a nutshell: inflation, bounce and late-time evolution. Phys. Rep. 692, 1-104 (2017). arXiv:1705.11098 [gr-qc]

11. E.J. Copeland, M. Sami, S. Tsujikawa, Dynamics of dark energy. Int. J. Mod. Phys. D 15, 1753-1936 (2006). arXiv:hep-th/0603057

12. G.W. Horndeski, Second-order scalar-tensor field equations in a four-dimensional space. Int. J. Theor. Phys. 10, 363-384 (1974)

13. C. Deffayet, X. Gao, D.A. Steer, G. Zahariade, From k-essence to generalised Galileons. Phys. Rev. D 84, 064039 (2011). arXiv:1103.3260 [hep-th]

14. C. Wetterich, Cosmology and the fate of dilatation symmetry. Nucl. Phys. B 302, 668-696 (1988). arXiv:1711.03844 [hep-th]

15. B. Ratra, P.J.E. Peebles, Cosmological consequences of a rolling homogeneous scalar field. Phys. Rev. D 37, 3406 (1988)

16. R.R. Caldwell, R. Dave, P.J. Steinhardt, Cosmological imprint of an energy component with general equation of state. Phys. Rev. Lett. 80, 1582-1585 (1998). arXiv:astro-ph/9708069

17. I. Zlatev, L.M. Wang, P.J. Steinhardt, Quintessence, cosmic coincidence, and the cosmological constant. Phys. Rev. Lett. 82, 896899 (1999). arXiv:astro-ph/9807002

18. C. Armendariz-Picon, T. Damour, V.F. Mukhanov, k-inflation. Phys. Lett. B 458, 209-218 (1999). arXiv:hep-th/9904075

19. C. Armendariz-Picon, V.F. Mukhanov, P.J. Steinhardt, A dynamical solution to the problem of a small cosmological constant and late time cosmic acceleration. Phys. Rev. Lett. 85, 4438-4441 (2000). arXiv:astro-ph/0004134

20. C. Armendariz-Picon, V.F. Mukhanov, P.J. Steinhardt, Essentials of $\mathrm{k}$ essence. Phys. Rev. D 63, 103510 (2001). arXiv:astro-ph/0006373

21. M.C. Bento, O. Bertolami, A.A. Sen, Generalized Chaplygin gas, accelerated expansion and dark energy matter unification. Phys. Rev. D 66, 043507 (2002). arXiv:gr-qc/0202064

22. K. Arun, S.B. Gudennavar, C. Sivaram, Dark matter, dark energy, and alternate models: a review. Adv. Space Res. 60, 166-186 (2017). arXiv:1704.06155 [physics.gen-ph] 
23. A.G. Cohen, D.B. Kaplan, A.E. Nelson, Effective field theory, black holes, and the cosmological constant. Phys. Rev. Lett. 82, 4971-4974 (1999). arXiv:hep-th/9803132

24. A.N. Tawfik, E.A. El Dahab, Review on dark energy models. Gravit. Cosmol. 25(2), 103-115 (2019)

25. G.J. Olmo, Palatini approach to modified gravity: $f(R)$ theories and beyond. Int. J. Mod. Phys. D 20, 413-462 (2011). arXiv:1101.3864 [gr-qc]

26. T. Harko, T.S. Koivisto, F.S.N. Lobo, G.J. Olmo, Metric-Palatini gravity unifying local constraints and late-time cosmic acceleration. Phys. Rev. D 85, 084016 (2012). arXiv:1110.1049 [gr-qc]

27. S. Capozziello, S. Tsujikawa, Solar system and equivalence principle constraints on $f(R)$ gravity by chameleon approach. Phys. Rev. D 77, 107501 (2008). arXiv:0712.2268 [gr-qc]

28. J. Khoury, A. Weltman, Chameleon cosmology. Phys. Rev. D 69, 044026 (2004). arXiv:astro-ph/0309411

29. N.A. Lima, Dynamics of linear perturbations in the hybrid metric-Palatini gravity. Phys. Rev. D 89(8), 083527 (2014). arXiv: 1402.4458 [astro-ph.CO]

30. N.A. Lima, V.S. Barreto, Constraints on hybrid metric-Palatini gravity from background evolution. Astrophys. J. 818(2), 186 (2016). arXiv:1501.05786 [astro-ph.CO]

31. S. Capozziello, T. Harko, T.S. Koivisto, F.S.N. Lobo, G.J. Olmo, Hybrid metric-Palatini gravity. Universe 1(2), 199-238 (2015). arXiv: 1508.04641 [gr-qc]

32. T. Harko, F.S.N. Lobo, Extensions of $f(R)$ Gravity: CurvatureMatter Couplings and Hybrid Metric-Palatini Theory. Cambridge Monographs on Mathematical Physics (Cambridge University Press, Cambridge, 2018)

33. T. Harko, F.S.N. Lobo, Beyond Einstein's general relativity: Hybrid metric-Palatini gravity and curvature-matter couplings. Int. J. Mod. Phys. D 29(13), 2030008(2020). arXiv:2007.15345 [gr-qc]

34. S. Capozziello, T. Harko, T.S. Koivisto, F.S.N. Lobo, G.J. Olmo, The virial theorem and the dark matter problem in hybrid metric-Palatini gravity. JCAP 07, 024 (2013). arXiv:1212.5817 [physics.gen-ph]

35. S. Capozziello, T. Harko, T.S. Koivisto, F.S.N. Lobo, G.J. Olmo, Galactic rotation curves in hybrid metric-Palatini gravity. Astropart. Phys. 50-52, 65-75 (2013). arXiv:1307.0752 [gr-qc]

36. S. Capozziello, T. Harko, F.S.N. Lobo, G.J. Olmo, Hybrid modified gravity unifying local tests, galactic dynamics and late-time cosmic acceleration. Int. J. Mod. Phys. D 22, 1342006 (2013). arXiv:1305.3756 [gr-qc]

37. S. Capozziello, T. Harko, T.S. Koivisto, F.S.N. Lobo, G.J. Olmo, Cosmology of hybrid metric-Palatini $\mathrm{f}(\mathrm{X})$-gravity. JCAP 04, 011 (2013). arXiv:1209.2895 [gr-qc]

38. S. Carloni, T. Koivisto, F.S.N. Lobo, Dynamical system analysis of hybrid metric-Palatini cosmologies. Phys. Rev. D 92(6), 064035 (2015). arXiv:1507.04306 [gr-qc]

39. S. Capozziello, T. Harko, T.S. Koivisto, F.S.N. Lobo, G.J. Olmo, Hybrid $f(R)$ theories, local constraints, and cosmic speedup. arXiv:1301.2209 [gr-qc]

40. C.G. Böhmer, F.S.N. Lobo, N. Tamanini, Einstein static Universe in hybrid metric-Palatini gravity. Phys. Rev. D 88(10), 104019 (2013). arXiv:1305.0025 [gr-qc]

41. Q.M. Fu, L. Zhao, B.M. Gu, K. Yang, Y.X. Liu, Hybrid metricPalatini brane system. Phys. Rev. D 94(2), 024020 (2016). arXiv: 1601.06546 [gr-qc]

42. J.L. Rosa, D.A. Ferreira, D. Bazeia, F.S.N. Lobo, Thick brane structures in generalized hybrid metric-Palatini gravity. Eur. Phys. J. C 81(1), 20 (2021). arXiv:2010.10074 [gr-qc]

43. K.A. Bronnikov, Spherically symmetric black holes and wormholes in hybrid metric-Palatini gravity. Gravit. Cosmol. 25, 331341 (2019). arXiv:1908.02012 [gr-qc]
44. C.Y. Chen, Y.H. Kung, P. Chen, Black hole perturbations and quasinormal modes in hybrid metric-palatini gravity. Phys. Rev. D 102, 124033 (2020). arXiv:2010.07202 [gr-qc]

45. J.L. Rosa, J.P.S. Lemos, F.S.N. Lobo, Stability of Kerr black holes in generalized hybrid metric-Palatini gravity. Phys. Rev. D 101, 044055 (2020). arXiv:2003.00090 [gr-qc]

46. K.A. Bronnikov, S.V. Bolokhov, M.V. Skvortsova, Hybrid metric-Palatini gravity: black holes, wormholes, singularities and instabilities. Gravit. Cosmol. 26(3), 212-227 (2020). arXiv:2006.00559 [gr-qc]

47. B. Danila, T. Harko, F.S.N. Lobo, M.K. Mak, Hybrid metric-Palatini stars. Phys. Rev. D 95(4), 044031 (2017). arXiv: 1608.02783 [gr-qc]

48. K.A. Bronnikov, S.V. Bolokhov, M.V. Skvortsova, Hybrid metricPalatini gravity: regular stringlike configurations. Universe 6, 172 (2020). arXiv:2009.03952 [gr-qc]

49. T. Harko, F.S.N. Lobo, H .M .R . da Silva, Cosmic stringlike objects in hybrid metric-Palatini gravity. Phys. Rev. D 101(12), 124050 (2020). arXiv:2003.09751 [gr-qc]

50. N. Avdeev, P. Dyadina, S. Labazova, Test of hybrid metric-Palatini $f(R)$-gravity in binary pulsars. J. Exp. Theor. Phys. 131(4), 537547 (2020). arXiv:2009.11156 [gr-qc]

51. J.L. Rosa, J.P.S. Lemos, F.S.N. Lobo, Wormholes in generalized hybrid metric-Palatini gravity obeying the matter null energy condition everywhere. Phys. Rev. D 98(6), 064054 (2018). arXiv: 1808.08975 [gr-qc]

52. S. Capozziello, T. Harko, T.S. Koivisto, F.S.N. Lobo, G.J. Olmo, Wormholes supported by hybrid metric-Palatini gravity. Phys. Rev. D 86, 127504 (2012). arXiv:1209.5862 [gr-qc]

53. M.S. Morris, K.S. Thorne, Wormholes in space-time and their use for interstellar travel: a tool for teaching general relativity. Am. J. Phys. 56, 395-412 (1988)

54. M.S. Morris, K.S. Thorne, U. Yurtsever, Wormholes, time machines, and the weak energy condition. Phys. Rev. Lett. 61, 1446-1449 (1988)

55. S. Kar, Evolving wormholes and the weak energy condition. Phys. Rev. D 49, 862-865 (1994)

56. S. Kar, D. Sahdev, Evolving Lorentzian wormholes. Phys. Rev. D 53, 722 (1996). arXiv:gr-qc/9506094

57. M. Visser, Lorentzian wormholes: from Einstein to Hawking (AIP Press, New York, 1995)

58. F.S.N. Lobo, Wormholes, warp drives and energy conditions. Fundam. Theor. Phys. 189 (Springer, Switzerland, 2017)

59. T.A. Roman, Inflating Lorentzian wormholes. Phys. Rev. D 47, 1370-1379 (1993). arXiv:gr-qc/9211012

60. L.A. Anchordoqui, D.F. Torres, M.L. Trobo, S.E. Perez Bergliaffa, Evolving wormhole geometries. Phys. Rev. D 57, 829-833 (1998). arXiv:gr-qc/9710026

61. A.V.B. Arellano, F.S.N. Lobo, Evolving wormhole geometries within nonlinear electrodynamics. Class. Quantum Gravity 23, 5811-5824 (2006). arXiv:gr-qc/0608003

62. E. Ebrahimi, N. Riazi, $(\mathrm{n}+1)$-Dimensional Lorentzian wormholes in an expanding cosmological background. Astrophys. Space Sci. 321, 217-223 (2009). arXiv:0905.3882 [hep-th]

63. E. Ebrahimi, N. Riazi, Expanding $(n+1)$-dimensional wormhole solutions in Brans-Dicke cosmology. Phys. Rev. D 81, 024036 (2010). arXiv:0905.4116 [hep-th]

64. M.R. Bordbar, N. Riazi, Time-dependent wormhole in an inhomogeneous spherically symmetric space time with a cosmological constant. Astrophys. Space Sci. 331, 315-320 (2011)

65. S.N. Sajadi, N. Riazi, Expanding Lorentzian wormholes in $R^{2}$ gravity. Prog. Theor. Phys. 126, 753-760 (2011)

66. M. Cataldo, F. Aróstica, S. Bahamonde, $(\mathrm{N}+1)$-dimensional Lorentzian evolving wormholes supported by polytropic matter. Eur. Phys. J. C 73(8), 2517 (2013). arXiv:1307.4122 [gr-qc] 
67. M.R. Setare, A. Sepehri, Role of higher-dimensional evolving wormholes in the formation of a big rip singularity. Phys. Rev. D 91(6), 063523 (2015). arXiv:1612.05077 [gr-qc]

68. S. Bhattacharya, S. Chakraborty, $f(R)$ gravity solutions for evolving wormholes. Eur. Phys. J. C 77(8), 558 (2017). arXiv: 1506.03968 [gr-qc]

69. A. Ovgün, Hawking's universe as an evolving dark wormhole. arXiv:1803.04256 [physics.gen-ph]

70. M. Cataldo, P. Labrana, S. del Campo, J. Crisostomo, P. Salgado, Evolving Lorentzian wormholes supported by phantom matter with constant state parameters. Phys. Rev. D 78, 104006 (2008). arXiv:0810.2715 [gr-qc]

71. M. Cataldo, S. del Campo, P. Minning, P. Salgado, Evolving Lorentzian wormholes supported by phantom matter and cosmological constant. Phys. Rev. D 79, 024005 (2009). arXiv:0812.4436 [gr-qc]

72. M. Cataldo, S. del Campo, Two-fluid evolving Lorentzian wormholes. Phys. Rev. D 85, 104010 (2012). arXiv:1204.0753 [gr-qc]

73. H. Maeda, T. Harada, B.J. Carr, Cosmological wormholes. Phys. Rev. D 79, 044034 (2009). arXiv:0901.1153 [gr-qc]

74. M.K. Zangeneh, F .S .N. Lobo, N. Riazi, Higher-dimensional evolving wormholes satisfying the null energy condition. Phys. Rev. D 90(2), 024072 (2014). arXiv:1406.5703 [gr-qc]

75. M. Kord Zangeneh, F.S.N. Lobo, H. Moradpour, Evolving traversable wormholes satisfying the energy conditions in the presence of pole dark energy. Phys. Dark Univ. 31, 100779 (2021). arXiv:2008.04013 [gr-qc]

76. M.R. Mehdizadeh, M. Kord Zangeneh, F.S.N. Lobo, EinsteinGauss-Bonnet traversable wormholes satisfying the weak energy condition. Phys. Rev. D 91(8), 084004 (2015). arXiv:1501.04773 [gr-qc]

77. M.R. Mehdizadeh, M. Kord Zangeneh, F.S.N. Lobo, Higherdimensional thin-shell wormholes in third-order Lovelock gravity. Phys. Rev. D 92(4), 044022 (2015). arXiv:1506.03427 [gr-qc]

78. M. Kord Zangeneh, F.S.N. Lobo, M.H. Dehghani, Traversable wormholes satisfying the weak energy condition in thirdorder Lovelock gravity. Phys. Rev. D 92(12), 124049 (2015). arXiv: 1510.07089 [gr-qc]

79. F. Parsaei, S. Rastgoo, Wormhole solutions with a polynomial equation-of-state and minimal violation of the null energy condition. Eur. Phys. J. C 80(5), 366 (2020). arXiv:1909.09899 [gr-qc]

80. S. Capozziello, F.S.N. Lobo, J.P. Mimoso, Energy conditions in modified gravity. Phys. Lett. B 730, 280-283 (2014). arXiv:1312.0784 [gr-qc]

81. S. Capozziello, F.S.N. Lobo, J.P. Mimoso, Generalized energy conditions in extended theories of gravity. Phys. Rev. D 91(12), 124019 (2015). arXiv:1407.7293 [gr-qc]

82. F.S.N. Lobo, A general class of braneworld wormholes. Phys. Rev. D 75, 064027 (2007). arXiv:gr-qc/0701133

83. F.S.N. Lobo, M.A. Oliveira, Wormhole geometries in $f(R)$ modified theories of gravity. Phys. Rev. D 80, 104012 (2009). arXiv:0909.5539 [gr-qc]

84. N.M. Garcia, F.S.N. Lobo, Wormhole geometries supported by a nonminimal curvature-matter coupling. Phys. Rev. D 82, 104018 (2010). arXiv:1007.3040 [gr-qc]

85. N. Montelongo Garcia, F.S.N. Lobo, Nonminimal curvaturematter coupled wormholes with matter satisfying the null energy condition. Class. Quantum Gravity 28, 085018 (2011). arXiv:1012.2443 [gr-qc]

86. T. Harko, F.S.N. Lobo, M.K. Mak, S.V. Sushkov, Modified-gravity wormholes without exotic matter. Phys. Rev. D 87(6), 067504 (2013). arXiv:1301.6878 [gr-qc]

87. R. Korolev, F.S.N. Lobo, S.V. Sushkov, General constraints on Horndeski wormhole throats. Phys. Rev. D 101(12), 124057 (2020). arXiv:2004.12382 [gr-qc]
88. G. Antoniou, A. Bakopoulos, P. Kanti, B. Kleihaus, J. Kunz, Novel Einstein-scalar-Gauss-Bonnet wormholes without exotic matter. Phys. Rev. D 101(2), 024033 (2020). arXiv:1904.13091 [hep-th]

89. T. Tangphati, A. Chatrabhuti, D. Samart, P. Channuie, Thin-shell wormholes in de Rham-Gabadadze-Tolley massive gravity. Eur. Phys. J. C 80(8), 722 (2020). arXiv:1912.12208 [gr-qc]

90. E. Papantonopoulos, C. Vlachos, Wormhole solutions in modified Brans-Dicke theory. Phys. Rev. D 101(6), 064025 (2020). arXiv: 1912.04005 [gr-qc]

91. A. Restuccia, F. Tello-Ortiz, A new class of $f(R)$-gravity model with wormhole solutions and cosmological properties. Eur. Phys. J. C 80(6), 580 (2020)

92. N. Godani, G.C. Samanta, Traversable wormholes in $R+\alpha R^{n}$ gravity. Eur. Phys. J. C 80(1), 30 (2020). arXiv:2001.00010 [grqc]

93. K.N. Singh, A. Banerjee, F. Rahaman, M.K. Jasim, Conformally symmetric traversable wormholes in modified teleparallel gravity. Phys. Rev. D 101(8), 084012 (2020). arXiv:2001.00816 [gr-qc]

94. A. Banerjee, M.K. Jasim, S.G. Ghosh, Traversable wormholes in $f(R, T)$ gravity satisfying the null energy condition with isotropic pressure. arXiv:2003.01545 [gr-qc]

95. T. Tangphati, A. Chatrabhuti, D. Samart, P. Channuie, Traversable wormholes in $f(R)$-massive gravity. Phys. Rev. D 102(8), 084026 (2020). arXiv:2003.01544 [gr-qc]

96. I. Fayyaz, M.F. Shamir, Wormhole structures in logarithmiccorrected $R^{2}$ gravity. Eur. Phys. J. C 80(5), 430 (2020). arXiv:2005.10023 [gr-qc]

97. M.G. Richarte, C. Simeone, Wormholes in Einstein-Born-Infeld theory. Phys. Rev. D 80, 104033 (2009). arXiv:2006.12272 [gr-qc] [Erratum: Phys. Rev. D 81, 109903 (2010)]

98. R. Ibadov, B. Kleihaus, J. Kunz, S. Murodov, Wormholes in Einstein-scalar-Gauss-Bonnet theories with a scalar selfinteraction potential. Phys. Rev. D 102(6), 064010 (2020). arXiv:2006.13008 [gr-qc]

99. B. Lazov, P. Nedkova, S. Yazadjiev, Uniqueness theorem for static phantom wormholes in Einstein-Maxwell-dilaton theory. Phys. Lett. B 778, 408 (2018). arXiv:1711.00290 [gr-qc]

100. A.A. Kirillov, E.P. Savelova, Wormhole as a possible accelerator of high-energy cosmic-ray particles. Eur. Phys. J. C 80, 45 (2020). arXiv: 1902.05742 [gr-qc]

101. D. Bak, C. Kim, S.H. Yi, Experimental probes of traversable wormholes. JHEP 12, 005 (2019). arXiv:1907.13465 [hep-th]

102. Z. Xu, M. Tang, G. Cao, S.N. Zhang, Possibility of traversable wormhole formation in the dark matter halo with istropic pressure. Eur. Phys. J. C 80, 70 (2020)

103. K. Jusufi, P. Channuie, M. Jamil, Traversable wormholes supported by GUP corrected Casimir energy. Eur. Phys. J. C 80, 127 (2020). arXiv:2002.01341 [gr-qc]

104. F.S.N. Lobo, A. Simpson, M. Visser, Dynamic thin-shell blackbounce traversable wormholes. Phys. Rev. D 101(12), 124035 (2020). arXiv:2003.09419 [gr-qc]

105. F. Parsaei, N. Riazi, Evolving wormhole in the braneworld scenario. Phys. Rev. D 102(4), 044003 (2020). arXiv:2004.01750 [gr-qc]

106. V. De Falco, E. Battista, S. Capozziello, M. De Laurentis, General relativistic Poynting-Robertson effect to diagnose wormholes existence: static and spherically symmetric case. Phys. Rev. D 101(10), 104037 (2020). arXiv:2004.14849 [gr-qc]

107. R. Moti, A. Shojai, Traversability of quantum improved wormhole solution. Phys. Rev. D 101(12), 124042 (2020). arXiv:2006.06190 [gr-qc]

108. T. Berry, F.S.N. Lobo, A. Simpson, M. Visser, Thin-shell traversable wormhole crafted from a regular black hole with asymptotically Minkowski core. Phys. Rev. D 102, 064054 (2020). arXiv:2008.07046 [gr-qc] 
109. M. Wielgus, J. Horak, F. Vincent, M. Abramowicz, Reflectionasymmetric wormholes and their double shadows. Phys. Rev. D 102(8), 084044 (2020). arXiv:2008.10130 [gr-qc]

110. S. Fallows, S.F. Ross, Making near-extremal wormholes traversable. JHEP 12, 044 (2020). arXiv:2008.07946 [hep-th]

111. J. Maldacena, A. Milekhin, Humanly traversable wormholes. Phys. Rev. D 103(6), 066007 (2021). arXiv:2008.06618 [hep-th]

112. F.S.N. Lobo, M. Visser, Fundamental limitations on 'warp drive' spacetimes. Class. Quantum Gravity 21, 5871-5892 (2004). arXiv:gr-qc/0406083

113. T. Harko, Z. Kovacs, F.S.N. Lobo, Electromagnetic signatures of thin accretion disks in wormhole geometries. Phys. Rev. D 78, 084005 (2008). arXiv:0808.3306 [gr-qc]
114. T. Harko, Z. Kovacs, F.S.N. Lobo, Thin accretion disks in stationary axisymmetric wormhole spacetimes. Phys. Rev. D 79, 064001 (2009). arXiv:0901.3926 [gr-qc]

115. T. Harko, Z. Kovacs, F.S.N. Lobo, Can accretion disk properties distinguish gravastars from black holes? Class. Quantum Gravity 26, 215006 (2009). arXiv:0905.1355 [gr-qc]

116. T. Harko, Z. Kovács, F.S.N. Lobo, Astrophysical signatures of thin accretion disks in wormhole spacetimes. Fundam. Theor. Phys. 189, 63-88 (2017) 